\title{
Regulation of expression level of fms-like tyrosine kinase- 4 is related to osteoclast differentiation
}

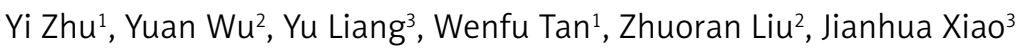

\author{
${ }^{1}$ Department of Orthopaedics, the Second Hospital Affiliated to the University \\ of South China, Hengyang, China \\ ${ }^{2}$ Department of Laboratory, the Second Hospital Affiliated to the University of South \\ China, Hengyang, China \\ ${ }^{3}$ Institute of Pathogen Biology, Medical College, the University of South China, \\ Hengyang, China
}

Submitted: 26 August 2013

Accepted: 18 October 2013

Arch Med Sci 2016; 12, 3: 502-506

DOI: 10.5114 /aoms.2015.55821

Copyright $\odot 2015$ Termedia \& Banach

\section{Abstract}

Introduction: The aim of this study is to determine whether regulation of the expression level of fms-like tyrosine kinase-4 (Flt-4) is related to osteoclast differentiation.

Material and methods: Osteoclast formation and differentiation of mouse bone marrow cells and RAW264.7 cells were performed. To induce osteoclast differentiation, RANKL $(50 \mathrm{ng} / \mathrm{ml})$ with or without vascular endothelial growth factor-C (VEGF-C) and vascular endothelial growth factor-D (VEGF-D) was added to mouse bone marrow cells and RAW264.7 cells. Then cells were examined under a microscope. TRAP-positive cells with 3 nuclei or more were considered as osteoclasts and counted. The Flt-4 gene was knocked down by transfection of siRNAs against Flt-4. Immunoblot analyses were performed.

Results: The osteoclast formation assay indicated that VEGF-C resulted in 500 or 450 vs. $100(p<0.05)$ of osteoclasts in mouse bone marrow cells and RAW264.7 cells, respectively. Vascular endothelial growth factor-D resulted in about 600 or 630 vs. 100 ( $p<0.05$ ) of osteoclasts for both mouse bone marrow cells and RAW264.7 cells. The knock-down of Flt-4 expression abolished the induction by VEGF-C or VEGF-D, resulting in induction similar to that of the negative control PBS.

Conclusions: Both VEGF-C and VEGF-D can induce osteoclast differentiation in the presence of the receptor activator of nuclear factor $\kappa B$ ligand. Down-regulation of expression level of Flt-4 protein abolishes osteoclast differentiation induced by VEGF-C or VEGF-D.

Key words: fms-like tyrosine kinase-4, osteoclast differentiation, vascular endothelial growth factor-C, vascular endothelial growth factor-D.

\section{Introduction}

Strength of the human skeleton is dependent on the balance between bone resorption by osteoclasts and bone formation by osteoblasts. In bone metabolisms, bone is resorbed by osteoclasts and replaced by new bone generated by osteoblasts [1]. Therefore, the balance between functions of osteoclasts and osteoblasts contributes to the maintenance of correct bone structure, bone mass and bone qualities. However, this important balance can be affected by pathological increases in osteoclast

\author{
Corresponding author: \\ Prof. Jianhua Xiao \\ Institute of Pathogen Biology \\ Medical College \\ the University \\ of South China \\ Hengyang, 421001, China \\ Phone: 86-734-8282907 \\ Fax: 86-734-8282907 \\ E-mail: jhxiao223@163.com
}


numbers, since it will lead to a net loss of bone. Osteoclasts are derived from pluripotent hematopoietic stem cells, which generate myeloid stem cells that then further differentiate into osteoclasts, megakaryocytes, granulocytes, and so on. Numerous hormones and cytokines generated in the bone microenvironment can regulate osteoclast differentiation and their functions [2-5]. The detailed mechanisms underlying regulation of osteoclast differentiation are not well understood but are of great clinical importance.

Vascular endothelial growth factors (VEGFs) are proteins produced by cells that stimulate vasculogenesis and angiogenesis. Vascular endothelial growth factors contributes to formation of new blood vessels during embryonic development or after injury. Vascular endothelial growth factors, including VEGF-A, -B, -C, -D, were found to be involved in many cellular processes [6-9]. Vascular endothelial growth factor-C and VEGF-D are ligands for the vascular endothelial growth factor receptor-3, which mediates lymphangiogenesis [10-14].

VEGF receptor-3 is an fms-like tyrosine kinase [15]; it is also termed fms-like tyrosine kinase-4 (Flt-4). Flt-4 is important for many signaling pathways. Interaction of Flt-4 and the focal adhesion kinase is related to survival of human neuroblastoma cell lines [16]. Mutations in Flt-4 result in Milroy disease, which is an autosomal dominant condition associated with congenital lymphedema [17]. In addition, it is reported that mutation in VEGF-C is associated with the autosomal dominant Milroy-like primary lymphedema [18].

In this study, we used VEGF-C and VEGF-D to induce osteoclast differentiation in cultured human peripheral blood mononuclear cells. We also used transfection of siRNA against Flt-4 to knock-down expression of Flt-4. It was found that decreases in expression of Flt-4 reduce osteoclast differentiation.

\section{Material and methods}

\section{Cell lines and reagents}

The mouse bone marrow cells and the macrophage/pre-osteoclast cell line RAW264.7 (purchased from ATCC, Manassas, VA, USA) were maintained in $\alpha$-minimal essential medium (MEM; Life Technologies, Grand Island, NY, USA), supplemented with $10 \%$ fetal bovine serum (FBS; CSL Biosciences, VIC, Australia), $0.005 \%$ penicillin $(10,000 \mathrm{U} / \mathrm{ml}) /$ streptomycin $(10,000 \mathrm{U} / \mathrm{ml})$ (Life Technologies, USA), L-glutamine (Life Technologies) and HEPES (Sigma-Aldrich, USA). Cells were cultured at $37^{\circ} \mathrm{C}$ with $5 \% \mathrm{CO}_{2}$ and $100 \%$ humidity. Recombinant soluble murine receptor activator of nuclear factor $\kappa \mathrm{B}$ ligand (RANKL) was purchased from the Oriental Yeast Co. (Tokyo, Japan). For tartrate resistant acid phosphatase (TRAP) staining, naphthol $A M X$ phosphate, fast red violet $L B$ Salt F-1625, and dimethylformamide were purchased from Sigma-Aldrich (St Louis, MO, USA). The human macrophage-colony stimulating factor (M-CSF) was purchased from R\&D Systems (Minneapolis, MN, USA). The rhVEGF-C and rhVEGF-D were purchased from R\&D Systems.

\section{Osteoclast differentiation}

Osteoclast formation and differentiation experiments were performed as previously described $[19,20]$. Briefly, mouse bone marrow cells $\left(1 \times 10^{5}\right.$ cells/well) and RAW264.7 cells $\left(1 \times 10^{4}\right.$ cells/well) were cultured in tissue culture wells $(6 \mathrm{~mm}$ diameter) containing $0.2 \mathrm{ml}$ of medium (MEM/FBS). To induce osteoclast differentiation, $50 \mathrm{ng} / \mathrm{ml}$ RANKL were added to the cells and the medium was replaced with fresh medium every 3 days. On day 8 of incubation, cells were fixed in $4 \%$ buffered formalin for 12 min and then washed with a mixture of methanol and acetone with a ratio of $1: 1$. The samples were air dried and histochemically stained for TRAP detection [21]. The TRAP-positive cells with 3 nuclei or more as examined by microscopy were considered as osteoclasts and counted.

For osteoclast survival assays, osteoclasts were dispersed using non-enzymic cell dispersion buffer (Sigma, cat. C5914, USA), rinsed with PBS, resuspended in MEM/10\% FBS and plated in $6 \mathrm{~mm}$ culture wells $\left(10^{5}\right.$ cells/well). Cells were cultured for $48 \mathrm{~h}$ with RANKL in combination with other indicated treatments (PBS, VEGF-C, VEGF-D, or M-CSF). Cells were then fixed and histochemically stained for TRAP examination and the osteoclast numbers were counted. Data were expressed as relative to positive control cultures stimulated by RANKL alone.

\section{siRNA experiments}

RAW264.7 cells were transfected with 80 pmol of siRNA against the mouse Flt-4 gene message (cat no. sc-35398; Santa Cruz Biotechnology) or a negative control siRNA (cat no. sc-37007, Santa Cruz Biotechnology) using X-tremeGENE (Roche, USA). After $4 \mathrm{~h}$, cells were then treated with Hsp90 inhibitors or vehicle control. Two days later, total protein was harvested, separated on $10 \%$ SDS/ PAGE gels, and subjected to immunoblot analyses.

\section{Immunoblot assays}

Total proteins were harvested from cells, separated on $10 \%$ SDS/PAGE gels, and then subjected to immunoblot analyses. The primary antibodies against Flt-4 and $\beta$-actin were purchased from Santa Cruz, USA (anti-Flt-4, cat \# sc- sc-365748, 1 : 200; anti- $\beta$-actin, cat\# sc-130301, $1: 10,000)$. Secondary antibodies were horseradish-peroxi- 


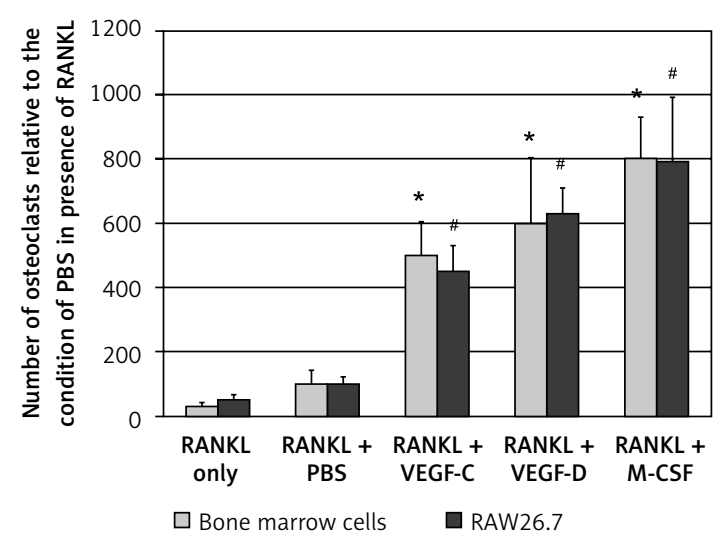

Figure 1. Effects of cytokines on osteoclast production. Osteoclasts of mouse bone marrow cells and RAW264.7 cells were cultured in the absence or presence of RANKL $(50 \mathrm{ng} / \mathrm{ml})$, together with PBS, VEGF-C (50 $\mathrm{ng} / \mathrm{ml})$, VEGF-D $(50 \mathrm{ng} / \mathrm{ml})$, or M-CSF $(30 \mathrm{ng} / \mathrm{ml})$. The number of osteoclasts in each condition was counted. All experiments were repeated at least 3 times

Data are expressed as means $\pm S D$. *Values of $p<0.05$, comparisons with RANKL only for osteoclasts of mouse bone marrow cells. "Values of $p<0.05$, comparisons with RANKL only for osteoclasts of RAW264.7 cells.

dase-conjugated secondary anti-mouse IgG (cat \# 31430, 1 : 10,000; Pierce Biotechnology). Bound antibodies were detected using the ECL system (Pierce Biotechnology, USA). The experiments were repeated at least 3 times. The mean normalized optical density (OD) of detected protein bands relative to the OD of the $\beta$-actin band was calculated.

\section{Statistical analysis}

The experimental data are expressed as mean + SD. Statistical software (SPSS 10.0, Chicago, USA) was used for independent sample $t$ tests. In all analyses, $p<0.05$ was considered statistically significant.

\section{Results}

\section{VEGF-C or VEGF-D induces osteoclast differentiation in the presence of RANKL}

The VEGF-C and VEGF-D are two growth factors that bind to Flt-4. To determine if VEGF-C or VEGF-D can induce osteoclast differentiation, the mouse bone marrow cells and RAW264.7 cells were cultured in the presence of RANKL, in the presence of VEGF-C, VEGF-D, or M-CSF. The M-CSF served as a positive control and PBS served as a negative control. On day 8 of incubation, cells were fixed in $4 \%$ buffered formalin and then washed with a mixture of methanol and acetone. TRAP-positive cells with 3 nuclei or more were considered as osteoclasts and counted as ratios of the negative control (PBS).
As shown in Figure 1, in the absence of RANKL, very few, if any, osteoclasts were produced. When compared with the condition in the presence of RANKL only (RANKL + PBS), VEGF-C resulted in 500 or 450 vs. $100(p<0.05)$ of osteoclasts of mouse bone marrow cells and RAW264.7 cells, respectively. VEGF-D resulted in about 600 or 630 vs. 100 ( $p<0.05$ ) of osteoclasts for both mouse bone marrow cells and RAW264.7 cells. Although lower than the effect of M-CSF on osteoclast induction, the effects of VEGF-C and VEGF-D were obvious (Figure 1). These results suggest that both VEGF-C and VEGF-D can induce osteoclast differentiation in the presence of RANKL.

\section{Knock-down of Flt-4 gene in pre-osteoclast RAW264.7 cells}

Since VEGF-C and VEGF-D bind to Flt-4 protein on the surface of cells, we further knocked down the Flt-4 gene in RAW264.7 cells. The cells were transfected with PBS only, the control siRNAs, or siRNA against Flt-4 gene using X-tremeGENE (Roche, USA). Two days later, total proteins were harvested, separated on 10\% SDS/PAGE gels, and subjected to immunoblot analyses. As shown in Figure 2, the levels of Flt-4 were significantly down-regulated in the RAW264.7 cells. These results suggest that the Flt-4 protein expression levels can be specifically knocked down in the RAW264.7 cells.

\section{Knock-down of Flt-4 protein abolishes the induction of osteoclast production by VEGF-C or VEGF-D}

Since we found that both VEGF-C and VEGF-D can induce osteoclast differentiation in the presence of RANKL, the related molecular mechanism was further investigated. The VEGF-C and VEGF-D both bind to Flt-4 protein on the surface of cells. We therefore determined the effects of knockdown of Flt-4 protein on the induction of osteoclast production by VEGF-C or VEGF-D. The Flt-4 gene siRNA-transfected RAW264.7 cells were cultured in the absence or presence of RANKL (50 ng/ $\mathrm{ml})$, together with PBS, VEGF-C (50 ng/ml), VEGF-D $(50 \mathrm{ng} / \mathrm{ml})$, or M-CSF $(30 \mathrm{ng} / \mathrm{ml})$. Eight days later, the number of osteoclasts in each condition was counted. All experiments were repeated at least 3 times. Data are expressed as means \pm SD.

As shown in Figure 3, when compared with the condition in the presence of RANKL only (RANKL $+\mathrm{PBS}), \mathrm{VEGF}-\mathrm{C}$ resulted in osteoclast production with a similar level to that of PBS only in RAW264.7 cells. The VEGF-D caused osteoclast production with a similar level to that of PBS only in RAW264.7 cells. However, the effect of M-CSF was still obvious, resulting in a 770 vs. 100 ( $p<0.05)$ when compared with the condition of PBS only. 
A

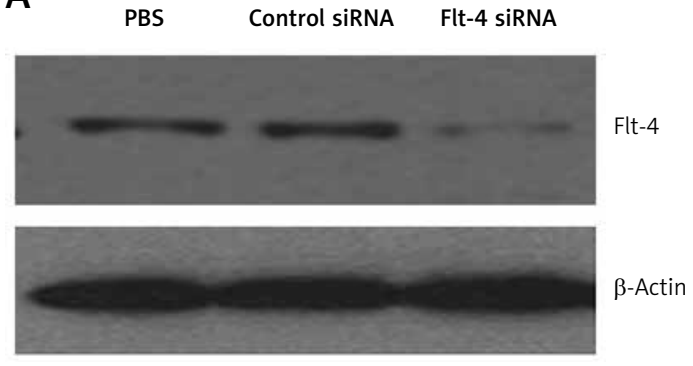

B

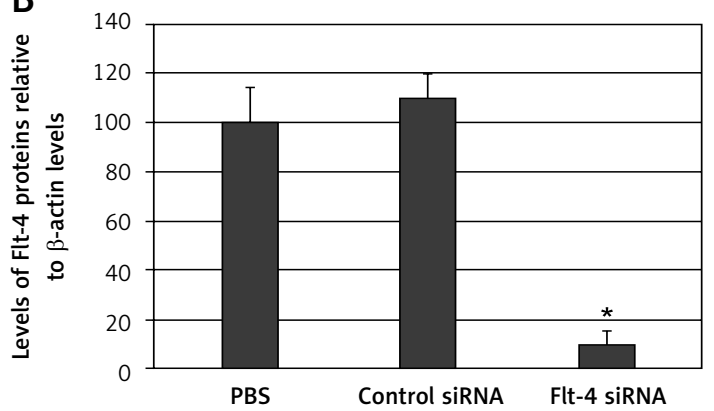

Figure 2. Knock-down of Flt-4 gene in RAW264.7 cells. A - RAW264.7 cells were transfected with PBS only, the control siRNAs, or siRNA against Flt-4 gene. RAW264.7 cells were transfected with 80 pmol of siRNA against the mouse Flt-4 gene message or a negative control siRNA using X-tremeGENE (Roche, USA). Two days later, total proteins were harvested, separated on $10 \%$ SDS/PAGE gels, and subjected to immunoblot analyses. B - The bands of Flt-4 proteins were scanned and the ratios (mean \pm SD) of the OD values relative to the OD value of $\beta$-actin bands were calculated

*Values of $p<0.05$, comparisons with cells transfected with the control siRNAs.

These results suggest that down-regulation of expression level of Flt-4 protein abolishes osteoclast differentiation induced by VEGF-C or VEGF-D.

\section{Discussion}

Many cellular factors were involved in the regulation of osteoclast differentiation and their functions [3-5, 22-24]. It have been reported that mutations of Flt-4 lead to Milroy disease, which is an autosomal dominant condition associated with congenital lymphedema $[16,17]$. In this study, we found that VEGF-C increased production of osteoclasts of mouse bone marrow cells and RAW264.7 cells. VEGF-D also resulted in about 600 or 630 vs. $100(p<0.05)$ of osteoclasts for both mouse bone marrow cells and RAW264.7 cells. Furthermore, the knock-down of Flt-4 abolished the induction by VEGF-C or VEGF-D, resulting in induction similar to that of the negative control PBS. Out findings suggest that down-regulation of the expression level of Flt-4 protein abolishes osteoclast differentiation induced by VEGF-C or VEGF-D.

Since VEGF-C and VEGF-D are important cellular factors involved in many cellular processes, our findings suggest that down-regulation of Flt-4 expression may affect other cellular processes induced by VEGF-C or VEGF-D. Recently, it was reported that VEGF-C and VEGF-D are involved in the inflammatory tumor microenvironment and inhibition of VEGF-C and VEGF-D delays the progression of skin tumors [25]. The serum VEGF-D level may act as a marker facilitating diagnosis of some disease such as lymphangioleiomyomatosis [26]. Therefore, our results suggest that Flt-4, VEGF-C, and VEGF-D might be used in prediction of development of some diseases.

In addition to Flt-4, many cellular proteins were found to be related to osteoclast differentiation. For example, the nuclear factor E2-related factor 2 affects RANKL-dependent osteoclastogenesis via

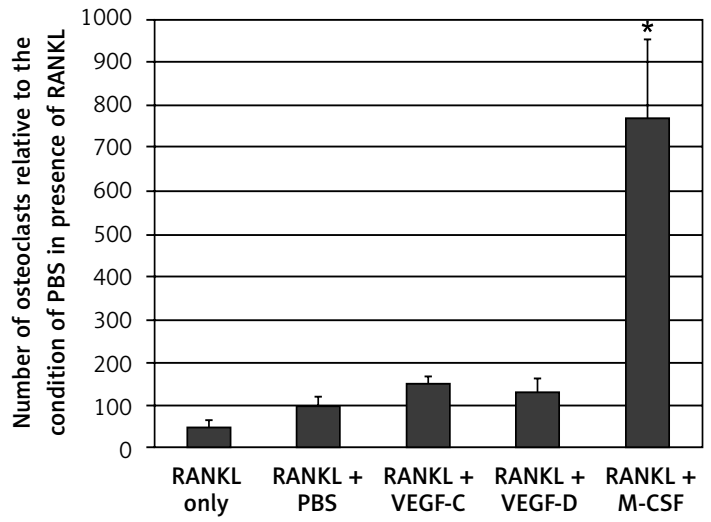

Figure 3. Effects of knock-down of Flt-4 gene on osteoclast production induced by VEGF-C and VEGF-D. The pre-osteoclast RAW264.7 cells were transfected with the control siRNAs or siRNA against the Flt-4 gene by the methods used in Figure 2. The Flt-4 gene siRNA-transfected RAW264.7 cells were cultured in the absence or presence of RANKL (50 ng/ $\mathrm{ml})$, together with PBS, VEGF-C (50 ng/ml), VEGF-D $(50 \mathrm{ng} / \mathrm{ml})$, or M-CSF $(30 \mathrm{ng} / \mathrm{ml})$. The number of osteoclasts in each condition was counted. All experiments were repeated at least 3 times

Data are expressed as means $\pm S D$. *Values of $p<0.05$, comparisons with RANKL only for osteoclasts of RAW264.7 cells.

alteration of intracellular ROS signaling through expression of cytoprotective enzymes [27]. Enhancement of the mitogen-activated protein kinase pathway and cyclooxygenase- 2 expression in RAW264.7 cells increases osteoclast differentiation and activation [28]. Therefore, the findings in this study provide new evidence for the cellular factor-mediated processes induced by VEGF-C [29, 30] and VEGF-D [31].

\section{Acknowledgments}

This work was supported by the Chinese National Natural Science Foundation (No. 81101274 , 
No. 30972576), Hunan Provincial Natural ScienceHengyang Joint Foundation (No. 10JJ2010), and Hunan Provincial Natural Science Foundation (No. 2011JJ3104).

\section{Conflict of interest}

The authors declare no conflict of interest.

\section{References}

1. Iqbal J, Sun L, Zaidi M. Coupling bone degradation to formation. Nat Med 2009; 15: 729-31.

2. Matsumoto Y, Otsuka F, Takano-Narazaki M, et al. Estrogen facilitates osteoblast differentiation by upregulating bone morphogenetic protein-4 signaling. Steroids 2013; 78: 513-20.

3. $\mathrm{Wu} \mathrm{H}$, Li L, Ma Y, et al. Regulation of selective PPARgamma modulators in the differentiation of osteoclasts. I Cell Biochem 2013; 114: 1969-77.

4. Kuo PL, Liao SH, Hung JY, Huang MS, Hsu YL. MicroRNA33a functions as a bone metastasis suppressor in lung cancer by targeting parathyroid hormone related protein. Biochim Biophys Acta 2013; 1830: 3756-66.

5. van Wijnen AJ, van de Peppel J, van Leeuwen JP, et al. microRNA functions in osteogenesis and dysfunctions in osteoporosis. Curr Osteoporos Rep 2013; 11: 72-82.

6. Reinert RB, Brissova M, Shostak A, et al. Vascular en dothelial growth factor-A and islet vascularization are necessary in developing, but not adult, pancreatic islets. Diabetes 2013; 62: 4154-64.

7. Lautenschlaeger T, George A, Klimowicz AC, et al. Bladder preservation therapy for muscle-invading bladder cancers on radiation therapy oncology group trials 8802 , 8903, 9506, and 9706: vascular endothelial growth factor B overexpression predicts for increased distant metastasis and shorter survival. Oncologist 2013; 18: 685-6.

8. Hofmann M, Pflanzer R, Zoller NN, et al. Vascular endothelial growth factor $\mathrm{C}$-induced lymphangiogenesis decreases tumor interstitial fluid pressure and tumor. Transl Oncol 2013; 6: 398-404.

9. Malinowska IA, Lee N, Kumar V, et al. Similar trends in serum VEGF-D levels and kidney angiomyolipoma responses with longer duration sirolimus treatment in adults with tuberous sclerosis. PLoS One 2013; 8: e56199.

10. Prangsaengtong O, Park JY, Inujima A, Igarashi Y, Shibahara N, Koizumi K. Enhancement of lymphangiogenesis in vitro via the regulations of HIF-1alpha expression and nuclear translocation by deoxyshikonin. Evid Based Complement Alternat Med 2013; 2013: 148297.

11. Pula B, Wojnar A, Witkiewicz W, Dziegiel P, Podhorska-Okolow M. Podoplanin expression in cancer-associated fibroblasts correlates with VEGF-C expression in cancer cells of invasive ductal breast carcinoma. Neoplasma 2013; 60: 516-24.

12. Matsumoto $M$, Roufail $S$, Inder $R$, et al. Signaling for lymphangiogenesis via VEGFR-3 is required for the early events of metastasis. Clin Exp Metastasis 2013; 30: 819-32.

13. Baluk P, Hogmalm A, Bry M, Alitalo K, Bry K, McDonald DM. Transgenic overexpression of interleukin-1beta induces persistent lymphangiogenesis but not angiogenesis in mouse airways. Am J Pathol 2013; 182: 1434-47.

14. Hirai S, Naito M, Terayama H, et al. Lymphangiogenesis in chronic inflammation in the testis. Andrology 2013; 1: 147-54.
15. Chappell JC, Mouillesseaux KP, Bautch VL. Flt-1 (vascular endothelial growth factor receptor-1) is essential for the vascular endothelial growth factor-notch feedback loop during angiogenesis. Arterioscler Thromb Vasc Biol 2013; 33: 1952-9.

16. Beierle EA, Ma X, Stewart JE, Megison M, Cance WG, Kurenova EV. Inhibition of the focal adhesion kinase and vascular endothelial growth factor receptor-3 interaction leads to decreased survival in human neuroblastoma cell lines. Mol Carcinog 2014; 53: 230-42.

17. Ghalamkarpour A, Holnthoner W, Saharinen P, et al. Recessive primary congenital lymphoedema caused by a VEGFR3 mutation. J Med Genet 2009; 46: 399-404.

18. Gordon K, Schulte D, Brice G, et al. Mutation in vascular endothelial growth factor- $\mathrm{C}$, a ligand for vascular endothelial growth factor receptor-3, is associated with autosomal dominant milroy-like primary lymphedema. Circ Res 2013; 112: 956-60.

19. Yeung YG, Jubinsky PT, Sengupta A, Yeung DC, Stanley ER. Purification of the colony-stimulating factor 1 receptor and demonstration of its tyrosine kinase activity. Proc Natl Acad Sci USA 1987; 84: 1268-71.

20. Quinn JM, Whitty GA, Byrne RJ, Gillespie MT, Hamilton JA. The generation of highly enriched osteoclast-lineage cell populations. Bone 2002; 30: 164-70.

21. Quinn JM, Morfis M, Lam MH, et al. Calcitonin receptor antibodies in the identification of osteoclasts. Bone 1999; 25: 1-8.

22. Tian B, Qin A, Shao ZY, et al. OA-4 Inhibits osteoclast formation and bone resorption via suppressing RANKL induced P38 signaling pathway. Curr Med Chem 2014; 21: 641-9.

23. Baud'huin M, Duplomb L, Teletchea S, et al. Osteoprotegerin: multiple partners for multiple functions. Cytokine Growth Factor Rev 2013; 24: 401-9.

24. Fukuda A, Goto T, Kuroishi KN, et al. Hemokinin-1 competitively inhibits substance P-induced stimulation of osteoclast formation and function. Neuropeptides 2013; 47: 251-9.

25. Alitalo AK, Proulx ST, Karaman S, et al. VEGF-C and VEGF-D blockade inhibits inflammatory skin carcinogenesis. Cancer Res 2013; 73: 4212-21.

26. Xu KF, Zhang P, Tian X, et al. The role of vascular endothelial growth factor- $D$ in diagnosis of lymphangioleiomyomatosis (LAM). Respir Med 2013; 107: 263-8.

27. Kanzaki H, Shinohara F, Kajiya M, Kodama T. The Keap1/ Nrf2 axis plays a role in osteoclast differentiation by regulating intracellular ROS signaling. J Biol Chem 2013; 288: 23009-20.

28. Hou GQ, Guo C, Song GH, et al. Lipopolysaccharide (LPS) promotes osteoclast differentiation and activation by enhancing the MAPK pathway and COX-2 expression in RAW264.7 cells. Int J Mol Med 2013; 32: 503-10.

29. Chen Y, Xu Y, Zheng J. Effect of carbon dioxide pneumoperitoneum on human renal cell carcinoma proliferation and metastasis in an orthotropic xenograft nude mouse model. Arch Med Sci 2014; 10: 1041-6.

30. Hogendorf P, Durczyński A, Kumor A, Strzelczyk J. Pancreatic head carcinoma and vascular endothelial growth factor (VEGF-A) concentration in portal blood: its association with cancer grade, tumor size and probably poor prognosis. Arch Med Sci 2014; 10: 288-93.

31. Zhang X, Sun Z, Jiang H, Song X. Relationship between single nucleotide polymorphisms in the 3'-untranslated region of the vascular endothelial growth factor gene and susceptibility to diabetic peripheral neuropathy in China. Arch Med Sci 2014; 10: 1028-34. 